\title{
Dans les coulisses de La Fille d'Aristide
}

Le manuscrit théâtral et les Graffigny Papers sous l'œil de la critique génétique

\section{Christina Ionescu}

\section{(2) OpenEdition \\ 1 Journals}

\section{Édition électronique}

URL : http://journals.openedition.org/genesis/926

DOI : $10.4000 /$ genesis.926

ISSN : 2268-1590

\section{Éditeur :}

Presses universitaires de Paris Sorbonne (PUPS), Société internationale de génétique artistique littéraire et scientifique (SIGALES)

\section{Édition imprimée}

Date de publication : 10 avril 2012

Pagination : 83-95

ISBN : 978-2-84050-822-9

ISSN : 1167-5101

\section{Référence électronique}

Christina Ionescu, «Dans les coulisses de La Fille d'Aristide», Genesis [En ligne], 34 | 2012, mis en ligne le 10 avril 2014, consulté le 13 juin 2020. URL : http://journals.openedition.org/genesis/926 ; DOI : https://doi.org/10.4000/genesis.926 


\section{Dans les coulisses de La Fille d'Aristide: le manuscrit théâtral et les Graffigny Papers sous l'œil de la critique génétique}

Christina Ionescu

J ouée pour la première fois le 29 avril 1758 à la Comédie-Française, La Fille d'Aristide échoua lamentablement et fut retirée par son auteure après la quatrième représentation. Avant cette chute mémorable dans l'histoire du théâtre des Lumières, Mme de Graffigny était déjà célèbre et célébrée en tant qu'auteure du succès de librairie Lettres d'une Péruvienne $(1747$; 1752) et de la comédie larmoyante Cénie (1750). Ce fut un échec qui fit couler l'encre car il était inattendu : à l'époque, l'auteure de La Fille d'Aristide était aussi une salonnière engagée dans les débats de son temps, une lectrice passionnée et friande de nouveautés littéraires, ainsi qu'une théâtromane consacrée. Spectatrice assidue des théâtres parisiens, Mme de Graffigny envoyait souvent à ses correspondants des comptes rendus animés et parfois minutieux des spectacles à l'affiche, dans lesquels elle livrait des détails précieux sur les conditions de leur mise en scène, sur les stratégies de programmation employées par les comédiens, sur leurs fréquentation et réception par le public. À quoi donc attribuer cette chute qui précéda de quelques mois la mort d'une auteure couronnée de succès et dotée d'une véritable culture théâtrale ? La clef d'un mystère parmi les plus spectaculaires dans le théâtre de l'Ancien Régime se cache dans la longue genèse de cette pièce - genèse intéressante en soi, certes, mais aussi pour ce qu'elle apprend au généticien sur les pratiques d'écriture et de composition d'une œuvre théâtrale conçue, textualisée, réinventée et représentée pendant une décennie charnière dans l'histoire du théâtre, les années 1750 , et pour ce qu'elle lui dévoile en plus à l'égard des mécanismes socioculturels régissant la production d'une dramaturge du siècle des Lumières. Le dossier génétique de La Fille d'Aristide, partie intégrante des manuscrits de $\mathrm{M}^{\mathrm{me}}$ de Graffigny que sa correspondance complète et éclaire, contient en plus quelques traces lointaines et passagères de la projection scénique du texte à l'état naissant, traces qui pourraient s'avérer utiles à une éventuelle génétique théâtrale propre au XVIII siècle, conçue sur les principes établis dans un ouvrage collectif paru tout récemment, Genèses théâtrales (2010), dont l'objectif est de poser les jalons et tracer les lignes directrices d'un champ de recherche qui « se situe en fait entre l'œuvre, l'opera, au sens latin du terme, désignant le travail préparatoire dans la durée, le soin, l'attention, la peine, les "répétitions" du faire théâtral et l'opus, terme renvoyant au travail achevé, mais jamais véritablement parachevé, puisque toujours soumis à des infléchissements dans la suite des représentations et des reprises possibles dans d'autres temps et d'autres conditions ${ }^{1}{ }^{»}$.

\section{L'intérêt des Graffigny Papers pour le généticien}

L'étude de l'ensemble des manuscrits liés à une œuvre littéraire commence par un travail préliminaire de génétique textuelle dont l'objectif principal est l'établissement et le classement du dossier. Lorsqu'ils furent sortis des archives familiales et survécurent au passage du temps, les manuscrits relatifs à une œuvre du siècle des Lumières sont plus susceptibles de se trouver aujourd'hui dispersés dans des collections publiques et privées en France ou à l'étranger. Dans le cadre du Projet Graffigny de l'Université de Toronto, qui vise la publication de la correspondance de l'écrivaine avec son ami lorrain et correspondant principal François-

1. Jean-Marie Thomasseau, « Pour une génétique du théâtre non contemporain : traces, objets, méthodes », dans Genèses théâtrales, Paris, CNRS Éditions, 2010, p. 233 ; voir aussi, dans le même ouvrage, Almuth Grésillon, Marie-Madeleine Mervant-Roux et Dominique Budor, « Pour une génétique théâtrale : prémisses et enjeux », p. 5-23. 
Antoine Devaux (1712-1796)2 ${ }^{2}$ les éditeurs ont envoyé des lettres aux directeurs des archives d'environ neuf cents bibliothèques afin de répertorier tous les documents autographes qui sont parvenus jusqu'à nous ; il n'est pas exclu, bien entendu, que d'autres manuscrits existants n'aient pas encore été repérés ${ }^{3}$. D'autres recherches de long terme menées par English Showalter et le reste de l'équipe éditoriale de la correspondance dirigée par J. A. Dainard nous permettent d'établir le parcours et d'indiquer la localisation actuelle de la plupart des documents conservés par Mme de Graffigny pendant sa vie ${ }^{4}$. Des mains des descendants de Devaux, la plupart des manuscrits et archives personnelles de l'auteure passèrent au XIXe siècle entre celles de deux collectionneurs étrangers. Une partie sans doute infime des Graffigny Papers fut acquise par le comte russe Grigorii Vladimirovich Orloff (favori de Catherine II, mieux connu en France comme Grégoire Orlov), qui rassembla lors de ses séjours à Paris une large collection d'autographes. La partie la plus importante fut pourtant achetée aux héritiers dans les années 1820 par sir Thomas Phillipps, figure imposante parmi les collectionneurs de son époque. Après la mort de Phillipps, son impressionnante collection fut liquidée aux enchères au cours de nombreuses ventes, les Graffigny Papers réapparaissant en juin 1965 à la succursale londonienne de Sotheby. Les cent un volumes originaux gardés dans leur reliure en carton ont été distribués comme suit : vingt-trois volumes, à la Bibliothèque nationale de France ; soixante-dix-huit volumes, à Hans Peter Kraus. Ce dernier, sans conteste l'antiquaire et bibliophile new-yorkais le plus prospère de la seconde moitié du Xxe siècle, en a fait don à la Beinecke Rare Book and Manuscript Library de l'université Yale aux États-Unis en 1968 (GEN MSS 353), mais il a gardé deux volumes $(6$ : les fameuses lettres de Cirey ; 96 : des manuscrits littéraires), volumes achetés par la Pierpont Morgan Library de New York en 1980. D'autres documents ayant trait à $\mathrm{M}^{\mathrm{me}}$ de Graffigny se trouvent actuellement dans le fonds Orlov ( ${ }^{\circ} 166$ du Département des sources écrites du Musée historique à Moscou) et dans les archives de la famille Duroselle.

Si l'évidence bibliographique indique clairement que, après sa mort et jusqu'au XIXe siècle où sa popularité s'éclipsa, Mme de Graffigny fut surtout connue comme romancière, et que, de nos jours pour la critique contemporaine (et surtout les féministes nordaméricaines), elle demeure toujours l'auteure des Lettres d'une Péruvienne, bien que la prise en connaissance de sa correspondance l'ait transformée petit à petit en la Sévigné de son temps, l'inventaire et le classement des Graffigny Papers ont comme résultat immédiat la peinture d'un autre portrait, vraisemblablement plus véridique, celui d'une dramaturge aux projets théâtraux foisonnants dès les années 1730 , époque de ses premières excursions dans le monde des belles-lettres en Lorraine, et jusqu'à la dernière décennie de sa vie, où elle se consacra pleinement à l'écriture et qui s'acheva sur l'échec paradoxal de La Fille d'Aristide. Outre la valeur philologique de tous ces manuscrits pour une éventuelle édition des œuvres complètes de $\mathrm{M}^{\mathrm{me}}$ de Graffigny, édition toujours à faire mais déjà envisagée par les amis de l'écrivaine dans les années qui suivirent sa mort, il faut souligner leur importance pour le généticien. En premier lieu, ils permettent d'apercevoir le processus créatif et la démarche scripturale diachroniquement, sur une période d'activité d'environ trente ans, au cours de laquelle l'auteure atteint le zénith de la créativité entre 1744, année de son début littéraire dans la capitale, et 1752 , date de la parution de la seconde édition autorisée des Lettres d'une Péruvienne. En deuxième lieu, ces manuscrits présentent un panorama du théâtre des années 1750 , montrant chez Mme de Graffigny la naissance, le développement et les métamorphoses de

2. J. A. Dainard (dir.), Correspondance de Madame de Graffigny, Oxford, Voltaire Foundation, 1985-, treize volumes déjà parus sur les seize envisagés (le seizième, électronique uniquement, sera consacré à un index et à un correctif). Je tiens à remercier Penny Arthur, Alan Dainard, English Showalter et David Smith pour les renseignements utiles qu'ils m'ont fournis lors de la rédaction de cet article, et Aurélie Zygel-Basso, qui a eu la gentillesse de relire ces pages. Les références aux lettres, qui seront données entre parenthèses dans le texte, renverront au volume et à la page en question ; pour la partie toujours inédite, sera indiquée seulement la date de la lettre.

3. Lors d'une vente aux enchères gérée par Pierre Bergé et associés en mai 2011, un recueil ayant appartenu à Devaux et qui contient des pièces diverses en partie autographes a été acheté par le château de Lunéville. Le manuscrit contient aussi les vers relatifs à l'échec de La Fille d'Aristide adressés par Devaux à Mme de Graffigny.

4. Voir English Showalter, Françoise de Graffigny. Her Life and Works, Studies on Voltaire and the eighteenth century [SVEC], 2004:11, p. 335-339. 
plusieurs types de pièces (drame bourgeois, comédie larmoyante, pièce édifiante, comédie de caractère, tragicomédie grecque, entre autres). C'est à travers l'examen d'une série complexe d'opérations génétiques qui conduisent chaque projet littéraire d'un stade à un autre (exploratoire, documentaire, scénarique, rédactionnel, consultatif, performatif, etc.) que se voit élargie notre connaissance de l'écriture graffignienne et que l'on aperçoit en plus les marques laissées par les phénomènes de censure institutionnelle et personnelle. En troisième lieu, l'analyse génétique du corpus manuscrit dévoile une autotranstextualité spécifiquement graffignienne : par exemple, une pièce rejetée par les comédiens, abandonnée ou non achevée peut contenir le germe d'un nouveau projet, s'imposant ainsi comme génératrice d'idées nouvelles et porteuse d'invention. Thèmes, intrigues, situations et personnages types sont repris et réinventés dans un processus de recyclage, voire parfois d'autocitation, révélateur tant des préoccupations auctoriales que d'une conception mécanique, artisanale de la création. D'une richesse surprenante est la documentation de La Fille d'Aristide, documentation a priori fragmentaire mais qui se caractérise néanmoins par la variété typologique des manuscrits et par leur richesse génétique. Véritable laboratoire de l'écriture, traces d'un processus de création théâtrale emblématique des Lumières et d'une histoire transformationnelle qui aboutit à des résultats singuliers, ces manuscrits permettent de reconstituer le cheminement avant-textuel de La Fille d'Aristide et apportent un nouvel éclairage sur la pièce représentée par les comédiens. Explorés en conjonction avec la correspondance de Mme de Graffigny, ils exposent avec acuité les hésitations, projections et spéculations de l'auteure, tout en soulignant l'importance du processus collaboratif qui marque la genèse de la pièce.

\section{Étude de cas : La Fille d'Aristide}

\section{Du canevas à la première mise au net}

Retracer le processus de création qui aboutira à la version imprimée de La Fille d'Aristide n'est pas sans importance pour le généticien : cela conduit inévitablement à des observations dignes d'intérêt sur le manuscrit théâtral d'Ancien Régime. La Brioche est mentionnée pour la première fois dans la lettre du 9 décembre 1749, où Mme de Graffigny entretient son correspondant lorrain d' « un canevas » qu'elle vient de recevoir de son amie Jeanne Quinault (16991783), ex-actrice de la Comédie-Française depuis 1741 , instigatrice et animatrice de la Société du Bout-du-Banc, surnommée « Nicole » dans les lettres à Devaux : «Je te dirai donc que samedi la grande conversation que j'eus avec Nicole est sur une piece qu'elle me prie de refaire d'un bout a l'autre » (X:288). Mlle Quinault exige que ce «diamant dans du fumier » soit «decrass[é] » en secret et Mme de Graffigny n'en apprend la raison que l'année suivante : l'auteur de l'ébauche n'était probablement autre que le comte de Caylus, qui d'une manière semblable lui avait offert le canevas d' « une nouvelle espagnole » en 1745 ; celui-ci voulait sans doute créer un rôle qui mît en valeur le talent de «sa donzelle »(XI:181), l'actrice Mlle Gauthier. La pièce portera le titre plaisant de La Brioche pendant plusieurs années (une métaphore qui associe évidemment l'acte de création à la fabrication pâtissière). Ces premières démarches rappellent le mécanisme de production mis en place par une autre société littéraire, les Lazzistes, ancêtres du Bout-du-Banc : « les instigateurs » prenaient l'initiative d'un projet et intervenaient par la suite activement dans sa réalisation, dont étaient chargés les « exécutants » et les «figurants », mais tout se déroulait en secret ${ }^{5}$. En outre, ancré dans la conversation et l'espace du salon, ce processus de création basé sur la collaboration d'un certain nombre de participants, à l'origine de bien des projets entrepris dans le cadre des théâtres de société et ateliers littéraires sous l'Ancien Régime, n'a pas toujours laissé des traces aujourd'hui visibles ou facilement décodables sur les manuscrits de l'époque.

\footnotetext{
5. David Trott, «"Histoire et Recueil des Lazzis" : le fonctionnement des jeux de théâtre secrets à Paris en 1731-1732 », SVEC, n 319 (1994), p. 117-128. Voir aussi l'édition du manuscrit : Histoire et recueil des Lazzis, éd. Judith Curtis et David Trott, SVEC, n 338 (1996). De par sa nature d'histoire, ce manuscrit est un des rares à enregistrer l'aspect collaboratif de la production des divertissements de société.
} 
(Co-)instigatrice du projet, Mlle Quinault ne donne pas carte blanche à la future auteure des Lettres d'une Péruvienne et veille à suivre de près l'évolution de son projet au cours de leurs rencontres régulières. En fait, Mme de Graffigny précise qu'elle est rémunérée pour ce travail (X:300), ce qui accorde au manuscrit une valeur monétaire et le statut d'un ouvrage confectionné sur commande. Grâce à la correspondance de l'auteure, nous disposons dans ce cas des circonstances initiatrices et provisionnelles de l'entreprise. À l'instar de tous les projets ainsi conçus par les sociétés littéraires de l'Ancien Régime, le stade exploratoire est absent de ce processus. Il n'empêche que La Brioche passe par un stade de décision et de programmation initiales lorsque Mme de Graffigny fait la critique du canevas. Elle trouve que « [1]e sujet est neuf, joli » et qu'il y a « des traits partout qu['elle] conserver[a] bien », mais elle en juge défavorablement d'autres aspects : « l'absurdité du plan », « [1]a detestabilité du dialogue, coq-a-l'ane perpetuel », « le froid et l'ineptie du tout ensemble », les personnages qui « sont d'etranges machine » $(\mathrm{X}: 288)$. On soupçonne donc que le canevas n'était pas un simple plan de travail mais une ébauche, peut-être même une version achevée, de la pièce. Le discours analytique tenu par Mme de Graffigny à son propos montre que, tout comme les critiques de théâtre de son siècle, elle privilégie le narratif par rapport au théâtral, s'intéressant surtout au sujet, au développement des personnages, à la conduite de l'action et à l'enchaînement des dialogues.

La phase rédactionnelle commence peu après cette rencontre initiale et dure jusqu'au mois de novembre 1750 . Pendant cette période, Mme de Graffigny ne travaille que sporadiquement sur La Brioche, obligée qu'elle est de mener d'autres projets en même temps. Lire les références à $L a$ Brioche dans la correspondance, c'est avoir accès à la dimension temporelle de l'œuvre in statu nascendi, c'est entrer dans l'espace d'intériorité auctoriale où l'écriture est un phénomène observable. Si l'on consulte, par exemple, la lettre du 21 mars, où Mme de Graffigny informe Devaux que, à l'aide de Mlle Quinault, elle a « relu et arrangé bien des choses a sa piece » $(\mathrm{X}: 435)$, on s'aperçoit du haut degré de collaboration auquel est sujette l'écriture de La Brioche - cependant, l'article possessif renvoie à l'ancienne actrice et son emploi suggère que $\mathrm{M}^{\mathrm{me}}$ de Graffigny ne considère pas encore la pièce comme sienne. Le 5 juin, on apprend que Mlle Quinault la pousse vivement à achever La Brioche (X:540), ce qui indique que $\mathrm{M}^{\mathrm{me}}$ de Graffigny était chargée du travail de rédaction, mais ce n'est que douze jours plus tard que cette dernière mentionne l'achèvement d'un premier brouillon, ajoutant qu' « [i]l y aura surement bien des corections a faire » (X:558). Il s'ensuit que dans l'esprit de l'écrivaine, l'œuvre demeure à ce stade toujours ouverte à la réflexion, à la suggestion, à la transformation. Plus tard, $\mathrm{Mme}$ de Graffigny écrit : «Ce matin on m'a raporté La Brioche du copiste. Je me suis mise a corriger les fautes d'oubli afin de l'envoier vite a Nicole... » (X:561) Dans la lettre à Devaux du 7 août, Mme de Graffigny indique qu'elle continue à réviser La Brioche, destinée maintenant à être jouée pendant l'absence de la cour. À cette fin, La Brioche doit être acceptée par les comédiens bien avant le départ pour Fontainebleau, prévu pour octobre. Dans la même lettre, l'écrivaine précise qu' « [i]l n'y a plus que des details a embelir » (XI:79). En effet, le mois d'août de cette année est marqué par un travail intense sur le texte. Le 24 août, Mme de Graffigny constate que l'ébauche originale a subi des changements d'ordre fondamental : « Il ne reste du modelle qu'on m'a donné que les noms des acteurs, que j'aurois bien voulu changer, et le fond du sujet » (XI:104). Ces propos, qui soulignent les disparités entre le modèle et sa métamorphose, évoquent le souci délibéré de l'écrivaine de prendre ses distances d'avec ce qui ne lui appartient pas, de s'approprier l'œuvre sur laquelle elle est en train de travailler, d'attester l'originalité de sa création.

Incapable de juger elle-même de ce nouvel état achevé de La Brioche, Mme de Graffigny sollicite l'avis prompt et sincère de Devaux. Le 26 août, son correspondant lorrain lui envoie un commentaire détaillé, où il en loue prudemment le style mais critique sévèrement le contenu. L'une des remarques de Devaux, motivée par la transgression d'une norme générique, orientera le développement du personnage principal dans les versions ultérieures : «Lisette est une heroine du tout et par tout, mais on ne scait ni pourquoy, ni comment, ni qui elle est, ni par quel hazard elle pense d'une façon toute contraire a son état. Je vous avouë que je ne scais a 
quoy cela tend. Qu'est-ce qu'une comedie qui n'est que le triomphe d'une soubrette qui reste soubrette ? Je ne scais si c'est prevention contre la nouveauté de ce genre, mais je ne prevois pas qu'il puisse plaire ni affecter » (XI:119). Dotée d'un cœur vertueux et bienveillant grâce à l'éducation remarquable qu'elle a reçue, la soubrette Lisette gère les affaires de Géronte, orchestre le mariage de Lucile avec Damon et assure la fortune de Valère. Il est intéressant de noter qu'elle refuse de se marier et choisit d'être maîtresse de sa vie, tout comme Zilia, l'héroïne des Lettres d'une Péruvienne. Devaux conseille à Mme de Graffigny de s'inspirer de l'histoire de Marie-Anne Pajot, racontée d'abord par $\mathrm{M}$ me Du Noyer dans ses Lettres historiques et galantes de deux dames de condition (1704), afin de refondre cette intrigue problématique. Selon Devaux, Lisette devrait être la « fille de bons et honnestes bourgeois ruinés » qui serait poursuivie par un ancien soupirant, ce qui engendrerait des malentendus ; il s'agirait ainsi d' " une action qui a une fin, qui conduit a quelque chose, et qui peut etre theatrale »(XI:131). La réponse de Devaux arrivée, Mme de Graffigny se défend ainsi : «J'ai voulu cent fois rendre la soubrette interessante. Cette diable de Nicole n'a jamais voulu. $\mathrm{Tu}$ a pu voir son but : c'est de se peindre elle-meme, de faire voir que l'on peut penser noblement en roture. C'est son mot »(XI:117) ${ }^{6}$. En lisant cet échange, on saisit pour la première fois les difficultés auxquelles se heurte l'écrivaine au travail dans le cadre restrictif de cette collaboration littéraire, car elle vient à l'évidence de développer une conscience d'auteur à l'égard de cette pièce. Ce sont les contraintes qui lui sont imposées qui déterminent le parcours de l'œuvre et assignent des frontières nettes à la liberté d'invention et d'élaboration dont jouit idéalement un auteur.

Une nouvelle étape rédactionnelle commence au mois de septembre : Mme de Graffigny remanie, par exemple, le premier acte afin de mieux dépeindre sa soubrette : « Il me semble que cela constate l'etat et le caractere de Lisette, que cela fixe l'attention sur elle et la rend interessante » (XI:120). L'extrait souligne l'importance accordée par l'auteure au personnage féminin, qui restera au centre des modifications ultérieures. Elle sollicite maintenant l'avis du père jésuite canadien Joseph-Ignace Martel de Belleville, qui « est enchanté des details et indigné du fond » (XI:133).
Le 15 septembre, Mme de Graffigny déclare qu'elle essaie en vain de trouver un nouveau titre pour La Brioche et exprime son désespoir à l'égard de cette « piece d'intrigue dont le principal personnage ne sera qu'une servante » (XI:146). Le 6 octobre, elle annonce le refus de la pièce par les acteurs du Théâtre-Français, refus qui met un terme à la première phase rédactionnelle.

\section{De la version achevée de La Brioche à la phase prérédactionnelle de La Fille d'Aristide}

La dernière version de La Brioche est une pièce achevée et prête à la représentation, pièce moderne de par la présence d'une soubrette dans le rôle principal et pour son traitement de thèmes comme l'aliénation sociale et la condition des femmes. Une nouvelle étape dans la genèse textuelle de La Fille d'Aristide commence à l'automne 1750. Dans une lettre qui date du 30 octobre, Mme de Graffigny envisage d'écrire une nouvelle pièce basée sur La Brioche en apportant des modifications considérables à l'intrigue et à l'action : «J'en veux faire une piece de caractere en jetant le sujet par la fenetre et replaquant les details sur un autre fond » $(\mathrm{XI}: 212)$. Mais ce n'est qu'en août 1751 qu'elle montre une dernière version de La Brioche à son ami et protégé Antoine Bret, auteur de La Double Extravagance (1750), qui lui « donn[e] de tres bonnes idées pour la metre en bonne forme »(XII:75). Au mois d'octobre de la même année, toujours décidée à refondre La Brioche, elle en présente une copie à JeanBaptiste-Charles-François de Rocot de Grandval (acteur, dramaturge et sociétaire de la Comédie-Française), « qui ne peut pas imaginer qu['elle] en fasse quelque chose »(XII:138). La critique de Grandval décourage l'écrivaine, qui ne se remettra au travail qu'une année plus tard.

Le processus rédactionnel commence à la fin de l'été 1752 et durera à peu près un an et demi. Le 1er septembre 1752, Madame de Graffigny annonce son nouveau dessein : "Je me suis mise à ecrire mon nouveau plan de La Brioche, que je veux raccomoder et remettre en cinq actes. Il y a lontems que je ne pouvois

6. Sur Mlle Quinault et ses idées sur le théâtre, voir Judith Curtis, «Divine Thalie: The Career of Jeanne Quinault », SVEC, 2007:08. 
pas trouver mon affaire. J'y suis a peu pres. La route est taillée, les tranchée sont faites dans cette foret où je ne voiois goute »(XIII:23). Elle lit le commencement de la pièce à Mlle Quinault en octobre et celle-ci en est « on ne peut pas plus contante » (XIII:84) ; quoiqu'elle continue à collaborer avec Mme de Graffigny, l'ancienne actrice n'exercera plus un contrôle vigilant sur le développement de la pièce comme auparavant. $\mathrm{Au}$ mois de décembre, où elle semble éprouver un plaisir considérable à travailler sur sa comédie, $\mathrm{M}^{\mathrm{me}}$ de Graffigny en annonce le nouveau titre : Les Effets de la prévention, traduite du grec. L'écrivaine déclare également qu'il ne s'agit pas d'une comédie larmoyante mais « du comique pur », observant sur le manuscrit : «Je crois cette piece susceptible d'assés de comique pour n'etre pas du genre larmoyant, quoiqu'en plus d'un endroit elle doive être touchante jusques aux larmes, mais de la facon dont les scenes comiques sont distribuées, elles ne nuiront point a celles qui sont pathetiques » (G. P., lxxvii:127).

En mars 1753, Mme de Graffigny montre un brouillon des trois premiers actes à Mlle Quinault et elles s'occupent ensemble des « etrivieres »[corrections] (XIII:211). Le 29 mars, l'écrivaine avise Devaux que le quatrième acte est entamé et promet de lui envoyer, après la mise au net, son « grifonage » (XIII:226) pour avoir son opinion. À l'aide de Bret, elle révise la pièce en avril, remaniant l'amour de Phædria pour Chloé, qui ressemblait trop à la jalousie, et changeant l'ordre des scènes pour mieux faire alterner le comique et le pathétique. À Devaux, elle explique en détail les raisons pour lesquelles elle transpose le lieu dans la Grèce antique :

C'est que je ne peux pas metre une jeune gouvernante ou femme de chambre dans le cas où est Misis [réincarnation de Lisette] chez des gens de qualité ; ce n'est pas notre usage et je ne sais pas faire parler les bourgeois. Parce qu'il n'y a point de philosophe parmis nous comme Cleomene. Parce que je ne veux pas traiter les preventions du grand monde, que je ne connois pas assés en details pour en prendre les tons. Parce que je n'aurois pas pu etablir l'amour de Glaucé en faisant dire des messes de requiem a son amant, et que cette scene me plait. Enfin, parce que j'ai voulu me peindre dans le caractere de Cleomene (XIII:250).
À ce stade, elle décide également de simplifier l'intrigue et de mettre l'accent sur une question qui la préoccupe depuis longtemps, les préjugés sociaux à l'égard d'un individu né dans un état perçu comme inférieur ou bas. Il est indiqué sur le manuscrit :

Il suffit de dire icy que le but theatral et moral de cette comedie est de faire triompher Misis du mepris que sa condition lui faisoit partager avec tout ce qui subissoit l'esclavage, et des accusations calomnieuses que le despit et la jalousie avoient imaginé pour la perdre, que ce triomphe est l'hommage que la prévention et la haine rendront a sa vertu et a sa probité ; et la reconnoissance que ses ennemis sont forcés de convenir qu'ils luy doivent (G. P., lxxvii:12).

Si la correspondance n'enregistre pas tous les détails concernant le processus de transformation de La Brioche en Les Effets de la prévention car, au cours de cette période, le salon de l'écrivaine déborde de monde du matin au soir et elle est impliquée dans toutes sortes de projets, seuls les manuscrits portent la trace d'un travail assidu et persévérant qui suit de près les projections que l'auteure fait dans les documents préparatoires. Néanmoins, tous ses efforts débouchent sur une déception en août, lorsque Mlle Quinault et Charles Pinot Duclos « [mettent] en pouldre [s]a pauvre pi[e] ce greques »(XIII:372)7 ${ }^{7}$. Peu après, Mme de Graffigny soupçonne sa collaboratrice d'en avoir envoyé une copie à l'habitué du Bout-du-Banc et spécialiste de la parade Charles-Alexandre Salley, dont elle attend «un in-folio de comentaire qui ne [lui] feront [...] que tres peu d'impression »(XIII:376) ; l'écrivaine n'en accuse la réception que le 3 mai 1754 , lorsqu'elle planifie déjà une nouvelle pièce de théâtre basée sur Les Effets de la prévention.

\section{De l'avant-texte au texte : les phases génétiques} de La Fille d'Aristide

Dans les six dernières années, la correspondance de Mme de Graffigny est ralentie par des querelles,

7. Sur le rôle de conseiller littéraire que joua l'auteur des Confessions $d u$ comte de *** auprès de l'écrivaine, voir Carole Dornier et David Smith, «Duclos vu par Mme de Graffigny », SVEC, n 371 (1999), p. 221-256. 
interrompue par des voyages de Devaux à Paris et à Versailles, et rédigée souvent par des amis proches de l'auteure en raison de sa mauvaise santé. Par conséquent, les références à la troisième phase d'écriture de sa pièce ne sont pas nombreuses, mais elles nous permettent tout de même d'observer la reprise d'un processus créatif qui nous est maintenant familier. Le début de cette troisième phase date de l'hiver 1754, où Mme de Graffigny demande à Devaux de lire la dernière version des Effets de la prévention et de la montrer à leurs amis lorrains parce qu'elle s'est décidée de nouveau à la refaire et aimerait profiter de leurs conseils. Après avoir relu elle-même le premier acte, elle se plaint de sa «lengueur », de sa «platitude» et de son « froit» $(31$ janvier 1754). La réponse de Devaux ne nous est pas parvenue, mais d'après ce qu'observe Mme de Graffigny le 6 février, cette fois-ci, sa réaction ne paraît pas avoir été marquée par trop d'enthousiasme. L'écrivaine reconnaît pourtant que, telle qu'elle demeure à présent, sa pièce n'est pas sans défauts, mais elle est résolue à la réécrire en améliorant tant la forme que le fond. Elle entretient ainsi Devaux de son nouveau plan d'action :

Il n'y a qu'un article que je ne te passe pas. C'est celui du lieu de la scene. Reflechis donc sur la liberté que cela donne a la philosophie et au ton. À présent je te dirai en un seul mot ce que j'en veux faire. L'esclave sera une belle-mere, fille d'un fameux Grec mort en exil dont je pers le nom dans ce moment-ci. Tu vois, je crois, par là combien le ton de la piece en sera anobli, combien elle peut devenir interessante, car ce ne sera pas le caractere de Misis. Ce sera la meme piece et c'en sera une autre, car il n'en restera que tres peu de chose, et combien la prevention sera mieux fondée (6 mars 1754).

Athènes remplace ainsi Delos ; le personnage principal, Théonise, qui n'est autre que la fille du célèbre général et homme d'état athénien, Aristide le Juste, succède à Misis, dont le statut social s'accorde mal à la thématique centrale des Effets de la prévention. Le 13 mars, Mme de Graffigny révèle à Devaux l'intrigue de cette nouvelle pièce. En l'absence de références ponctuelles à La Fille d'Aristide dans la correspondance, la datation des manuscrits ne peut être qu'approximative pour la période 1754-1757. En fait, la référence suivante à cette pièce, qui date d'une année plus tard, nous apprend que, le 20 avril 1755, elle finit un nouveau plan et, le 8 juin, elle veut envoyer à Devaux le premier acte. Le quatrième est déjà achevé en octobre et elle se moque du manque d'intérêt que manifeste son ami à lire une pièce qui « devient plus interessante que jamais pour [elle] » (25 octobre 1755). Le 14 novembre, elle annonce que le cinquième acte est terminé et qu'elle vient de commencer les corrections. "La première revuë generalle » (28 novembre 1755) de Mlle Quinault n'a lieu que le 1er décembre : « $[\mathrm{H}]$ ier je fus enfermée avec Nicole tout le jours pour rabotter mon affaire. Nous avons à peu pres culbuté le cinq, recommencer deux scenes du 4, une du trois, et pas un mot au 1er ny au second. Elle en est toujours ravie. Elle a pleuré comme une bete... » À la fin de l'année, on la juge prête à la lecture de salon et les larmes qui coulent sont le garant de sa réussite : «Nicole est venue entendre Theonise, lue par Collé, et fondre en larmes »; on veut « la transcrire en pleine ligne pour la presenter a la coupelle de Duclos » (28 décembre 1755).

La correction de ce dernier jet se poursuit dans la première moitié de l'année 1756. Le 20 avril, Mme de Graffigny envoie à Devaux les brouillons des trois premiers actes corrigés. Pour la première fois, on voit que la révision effectuée par Mme de Graffigny, fondée à de nombreuses reprises sur les commentaires de ses amis, prend en compte la portée scénique de son texte et révèle son esthétique théâtrale. L'écrivaine remarque, par exemple, qu'elle préfère sacrifier la vraisemblance des événements à l'intensité de la situation représentée sur la scène. En ce qui concerne le premier acte, elle est prête à le raccourcir, mais ne voit pas la raison car «[c]'est un traité d'esprit et de moralle qui passe toujours » et « [i]l faut de furieux defauts dans un $1^{\mathrm{r}}$ acte pour etre hué » (30 avril 1756). Dans cette même lettre, inquiète du sort de sa pièce, elle tient Devaux au courant de l'état actuel du travail :

On copie le 4 et je finirai peut-etre demain le cinq. C'est celui-la qui me coute et où il faut peser les mots au poids de l'or, c'est celui-la pour lequel je te crains et plus encore le public. Tous mes amis le trouvent bien et moi je ne l'aime pas. À l'égard du 4e je me flate que tu diras comme Duclos que c'est le plus beau qui soit au theatre sans exception [...] mais je crains toujours que l'on ne prene le denouement pour une machine, quoi que ce soit l'histoire toute pure. 
Selon toute apparence, Mme de Graffigny considère comme achevée la version de La Fille d'Aristide de 1756. Par l'intermédiaire de Laugier, médecin d'origine lorraine établi à la cour impériale de Vienne, elle demande à l'impératrice Marie-Thérèse d'Autriche de recevoir la dédicace de La Fille d'Aristide. La pièce est présentée sans nom d'auteur aux acteurs de la Comédie-Française par Charles Collé qui leur en fait la lecture, mais les comédiens reconnaissent la plume de l'auteure. La représentation de La Fille d'Aristide a lieu à un moment décisif dans l'histoire du théâtre français, voire à l'aube même de l'émergence d'une nouvelle dramaturgie. Enracinée dans la genèse longue et compliquée de la pièce, cette chute représente tant l'échec d'une pratique d'écriture collaborative, si répandue dans les milieux littéraires de l'époque, que les déboires d'une transposition sur scène qui, à l'évidence, ne répond plus aux nouvelles exigences du public.

Aussi peut-on conclure que le processus d'écriture acharné mais épisodique de La Fille d'Aristide s'étend sur une période de neuf ans (1749-1758). Cette genèse est marquée par des interruptions dues à des avis décourageants des lecteurs et par des reprises obstinées de l'œuvre in progress, voire des retours quasi obsessionnels à un travail persévérant de réécriture et de relecture dont la trajectoire se dessine dans la correspondance de l'auteure. L'itinéraire scriptural est parsemé de moments de blocage et de réflexion de durée variable, de relances enthousiasmées de l'écriture, de retours en arrière et d'intégration d'idées nouvelles. Qui plus est, souvent cet acte d'écriture prend la forme d'un travail collectif sur l'œuvre aux stades de la conception, du développement, de la transformation. Les amis de Mme de Graffigny lui présentent leurs réactions face aux différentes étapes de prospection et de rédaction, en lui suggérant des modifications à effectuer et des pistes à suivre; de temps en temps, ils participent activement à la création de l'œuvre. On peut soupçonner que si la prospection scénique est souvent absente des manuscrits préparatoires, c'est parce qu'elle se fait oralement, dans les consultations avec Mlle Quinault. Sous-jacent à la production de l'œuvre se présente dans la correspondance de l'auteure tout un discours sur le siècle des Lumières, où l'œuvre littéraire est souvent le produit d'une collaboration et où les femmes auteurs dépendent souvent des conseils et de l'aide de leurs amis.

\section{Appendice : échantillon de documents génétiques}

Le parcours génétique de La Fille d'Aristide établi grâce à la correspondance nous permet de diviser les manuscrits en trois catégories principales, compte tenu du fait que les deux premiers états achevés correspondent chacun à une pièce complète et prête au passage sur scène (La Brioche et Les Effets de la prévention). Il s'agit d'un dossier riche et varié qui contient des plans, fragments, brouillons, jets successifs avec ou sans corrections ultérieures, mises au net, copies de la main d'un secrétaire ou copiste, critiques, etc. - dossier qui montre une dramaturge dont l'activité créatrice n'est pas mentale, qui a besoin du support écrit pour étaler ses pensées et développer ses idées. À propos de l'importance du plan, par exemple, on lit sur le manuscrit de travail : «C'est une erreur de croire qu'un plan refroidit le compositeur. Corneille qu'on ne peut en general accuser d'etre froid comptoit sa piece faite lorsqu'il etoit parvenu a faire son plan. Je crois qu'en travaillant sur un plan scene a scene sans y etre rempli la tête de l'arrangement total et de toutes les parties relatives de son intrigue et des situations qu'elle renferme on feroit chaque scene aussi languissament que des bouts rimés » (G. P., lxxvii:127-128). Plusieurs strates se superposent sur ces pages manuscrites : corrections autographes ou suggestions d'autres mains, réflexions métathéâtrales et commentaires critiques débordent parfois dans les marges, saturent souvent le texte ou s'ajoutent sous la forme de pages entières ou morceaux collés. Voici un échantillon (les références entre parenthèses renvoient aux Graffigny Papers) :

\section{La Brioche}

- Dernier état de La Brioche, de belle main, avec une particularité intéressante pour le travail du généticien : ce que Mme de Graffigny appelle des «emplâtres » (morceaux de papier collés contenant des révisions) 


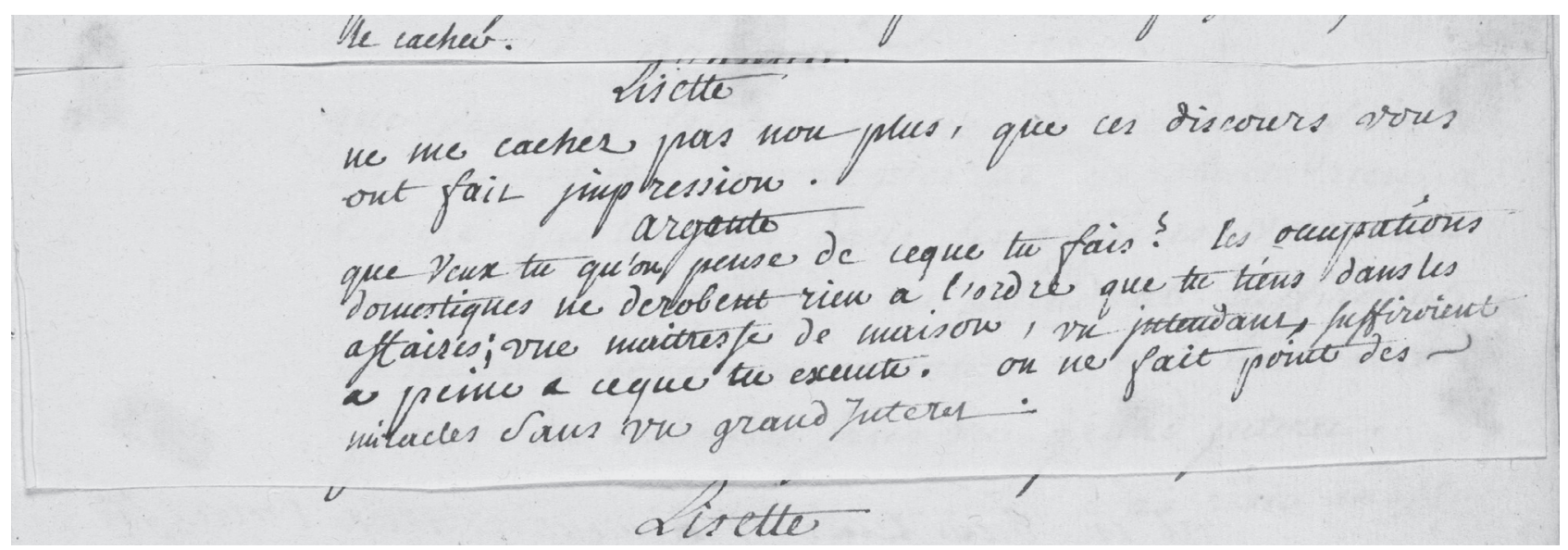

Fig. 1 : Extrait d'une page de La Brioche de la main d'un secrétaire avec un « emplâtre »,

morceau collé à l'original contenant des corrections (The Beinecke Rare Book and Manuscript Library, Graffigny Papers, lxxix:17)

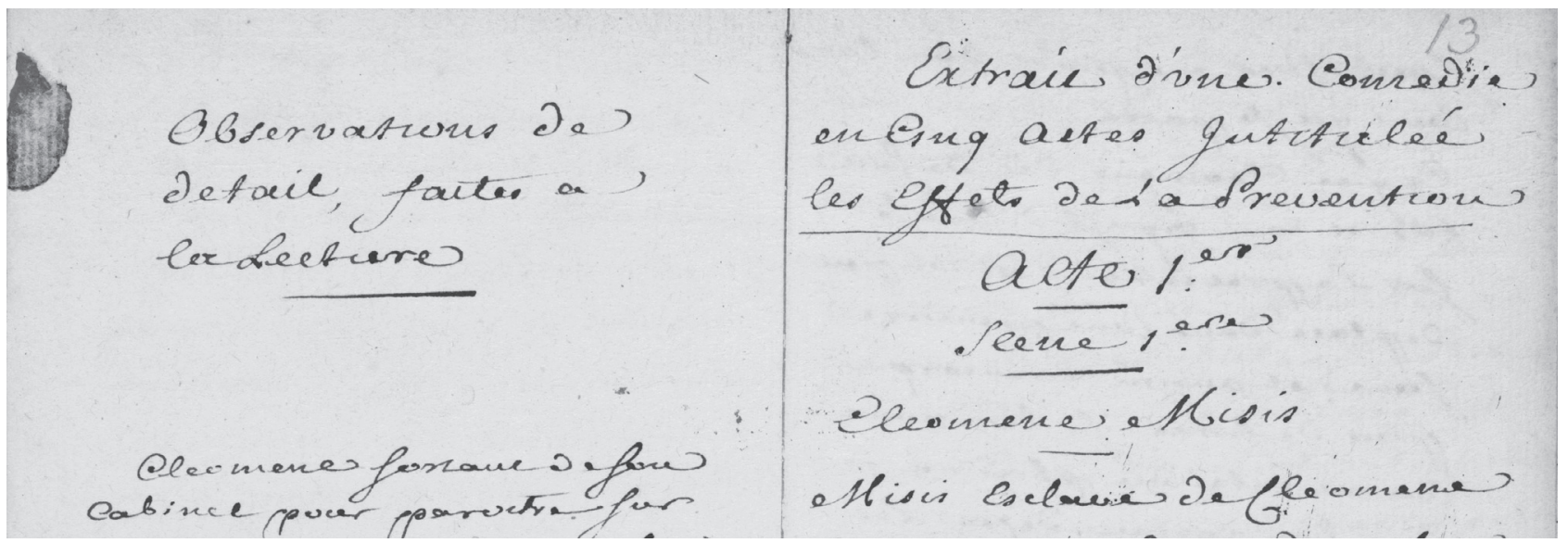

Fig. 2 : Début d'un état préparatoire des Effets de la prévention : à gauche, « Observations de detail, faites à la lecture » du plan ; à droite, « Extrait d'une comedie en cinq actes intitulée les Effets de la Prevention », un plan détaillé des scènes (The Beinecke Rare Book and Manuscript Library, Graffigny Papers, lxxvii:13)

(lxxix:1-97) (fig. 1); état antérieur au précédent, incomplet (lxxviii:165-254) ; fragments (lxxviii:81-84 et 267-278).

\section{Les Effets de la prévention}

- «Actions qui ont precedé la pièce et qui doivent etre rapellées dans l'exposition »(1xxvii:1-5; les pages du volume sont divisées par une ligne verticale, pour laisser de l'espace aux remarques à droite) ; rôles et lieu (lxxvii:6) ; caractérisation élaborée de chaque personnage, avec une clarification importante sur l'argument de la pièce à la fin (1xxvii:6-12) ; «Observations de detail, faites à la lecture » (à gauche) et « Extrait d'une comedie en cinq actes intitulée les Effets de la Prevention » (à droite), ce dernier étant un plan détaillé, scène par scène, inachevé (lxxvii:13-27, 28-36 pages blanches) (fig. 2) ; «Observations » (à gauche) et plan détaillé, complet (lxxvii:37-125); critiques générales (lxxvii:125-128) ; fragment autographe sur le caractère de Misis (lxxviii:106) ; version dialoguée, copie professionnelle, la moitié droite de la page laissée libre, sans ajouts (lxxx:1189) ; version dialoguée d'une belle main, avec des observations et corrections pour la plupart autographes (infrapaginales et emplâtres), titre de la main tardive de l'écrivaine (lxxxi:69-138) ; de nombreux fragments (dans les volumes lxxviii, xc, xcii et xcvii). 


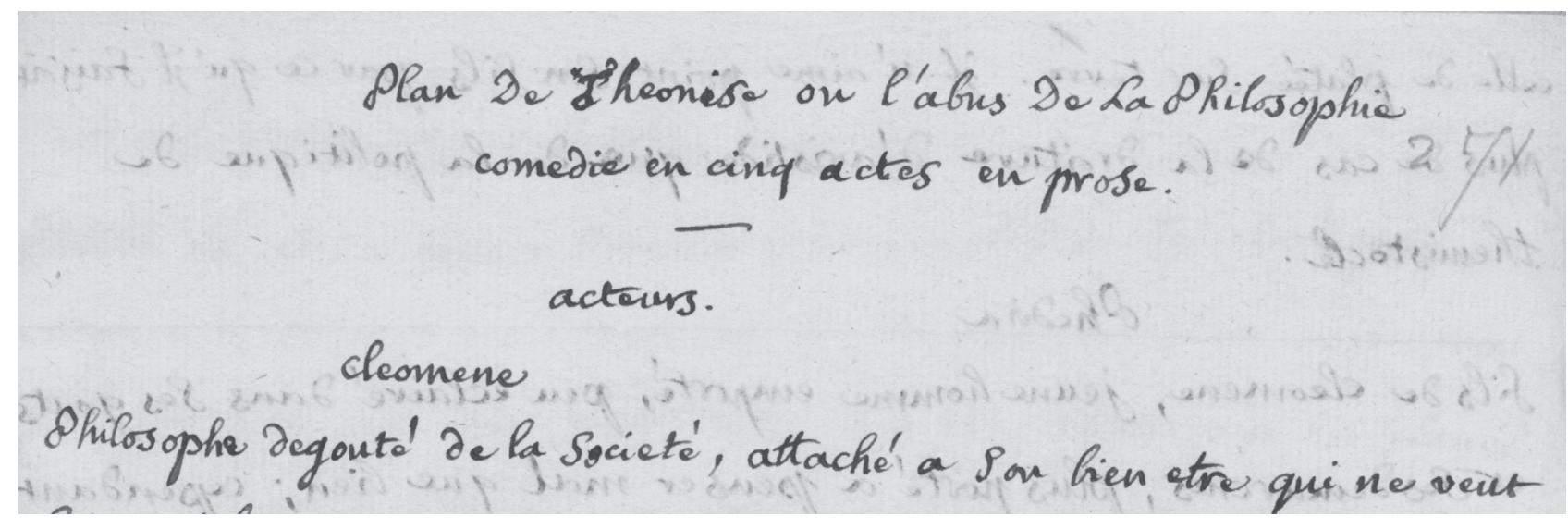

Fig. 3 : Extrait du «Plan de Theonise ou l'abus de la philosophie, comedie en cinq actes en prose », état préparatoire de La Fille d'Aristide, pièce intitulée provisoirement Théonise, nom de la fille d'Aristide et rôle principal (The Beinecke Rare Book and Manuscript Library, Graffigny Papers, xciii:271)

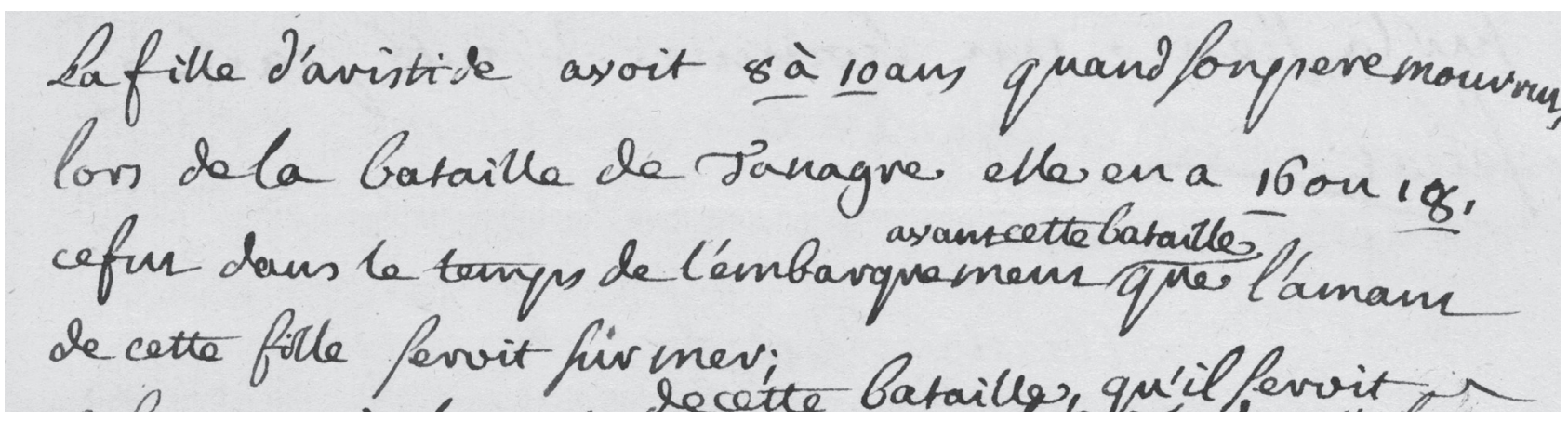

Fig. 4 : Remarque sur la fille d'Aristide, extraite d'un document préparatoire qui contient des notes de lecture à propos de l'histoire de l'homme d'État athénien du ve siècle av. J.-C., Aristide le Juste (The Beinecke Rare Book and Manuscript Library, Graffigny Papers, xcii:21)

\section{La Fille d'Aristide}

- Plusieurs états de La Fille d'Aristide : «Acteurs », description des personnages (lxxxi:153-154, sans doute la mise au net de lxxxi:187-188, qui contient des corrections de la main de l'auteure ; la version autographe de ce dernier est lxxxi:205-206) ; plan de chaque scène, avec quelques corrections autographes (lxxxi:155-172; le fragment classé sous lxxxi:189-190 est ici mis au net); fragment d'un autre plan, antérieur au précédent, avec de nombreux ajouts de la main de l'auteure (lxxxi:173-185) ; plan autographe (lxxxi:191-202), état mis au net dans lxxxi:173-185, qui n'est pas complet; « Plan de Theonise ou l'abus de la philosophie, comedie en cinq actes en prose », qui contient la description des rôles et l'intrigue, scène par scène (xciii:271-287) (fig. 3) ; fragments de diverses mains dans les G. P., trop nombreux à énumérer (dans les volumes lxxviii, lxxxi, xc et xcii);

- notes et réflexions sur l'histoire d'Aristide ( $\mathrm{Ce}$ fut vers l'an 466 avant l'Ere vulgaire que mourut Aristide... », xcii:19-28), avec un court paragraphe sur sa fille (« La fille d'Aristide avoit 8 à 10 ans quand son pere mourra lors de la bataille de Tanagre...», p. 21) (fig. 4) ;

- critique très intéressante de La Fille d'Aristide par Yves-Jean-Baptiste de la Boissière de Chambors (7 juillet 1755, BnF, NAF 15579, fos 11-16) ;

- une «scène contre la philosophie prétendue, et les prétendus philosophes », rédigée par Charles Collé pour La Fille d'Aristide, non utilisée 8 ;

- une copie de La Fille d'Aristide, écrite d'une belle main de copiste, qui contient les noms des acteurs (xci:128-251) : Théonise... Mlle Gaussin, Thaïs... Mlle Dangeville, Cléomène... Mr Sarrazin, Cratobule... Mr Préville, Trazile... Mr Grandval, Phérès... Mr Bellecour, Parménon... Mr Lanoüe, Dromon... Mr Armand (fig. 5). Ce manuscrit contient des instructions destinées au jeu des acteurs (exemple :

8. Charles Collé, Journal historique, ou Mémoires critiques et littéraires, Paris, Imprimerie bibliographique, 1807, t. II, p. 208-210. 
"Cléomène veut se jetter aux pieds de Théonise ", p. 116). Selon un document qui date de l'année 1759, les acteurs nommés Sarrazin et Lanoüe ne faisaient plus partie de la troupe ${ }^{9}$. Il semble presque certain que la distribution se rapporte à la représentation prévue pour 1757, avant les dernières corrections de l'auteure. Un état antérieur à celui-ci, toujours avec les noms des acteurs mais différemment orthographiés, se trouve dans xcvii:1127 ;

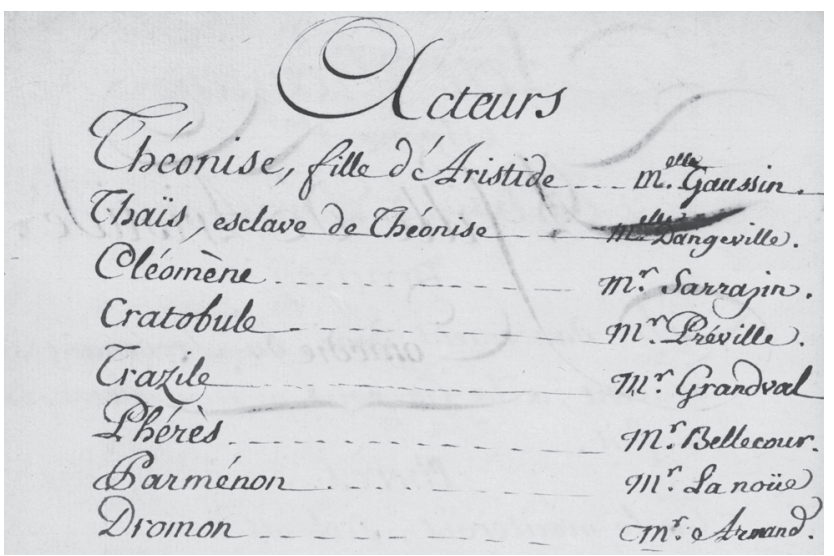

Fig. 5 : État achevé de La Fille d'Aristide, d'une belle main de copiste, avec les noms des acteurs de la Comédie-Française (The Beinecke Rare Book and Manuscript Library, Graffigny Papers, xci:130)

- fragments de la version de La Fille d'Aristide «pour souffler », d'une main de copiste, avec des corrections autographes (lxxxi:213-332) ; état fortement remanié, incomplet (fig. 6) ;

- l'un des états (xcvii:129-263) contient l'autorisation de représentation de la pièce, signée par Crébillon à la fin du dernier acte, sur la page du manuscrit, en bas de la dernière réplique de Cléomène : " $\mathrm{J}$ 'ay lu par ordre de Monsieur le Lieutenant Général de Police La Fille d'Aristide et je crois que l'on peut en permettre la représentation ce 28 avril 1758 Crébillon »; et puis, «Vu Permis de representer ce 28 avril 1758 Bernin». Ce manuscrit, de la main du copiste, a été corrigé par l'auteure. Il n'est pas identique à la version imprimée (fig. 7) ;

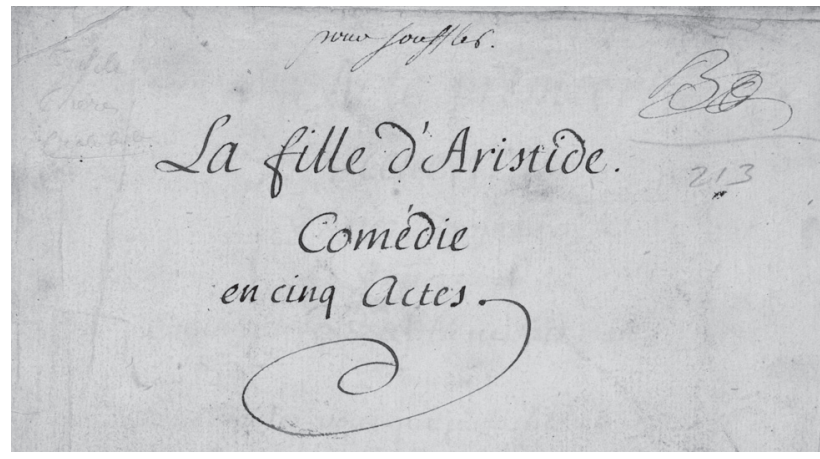

Fig. 6 : Page de titre d'un autre état de La Fille d'Aristide. La mention « pour souffler » indique qu'il s'agissait du manuscrit destiné au souffleur. Mme de Graffigny remania cet état avant la représentation de la pièce en 1758

(The Beinecke Rare Book and Manuscript Library, Graffigny Papers, lxxxi:213)

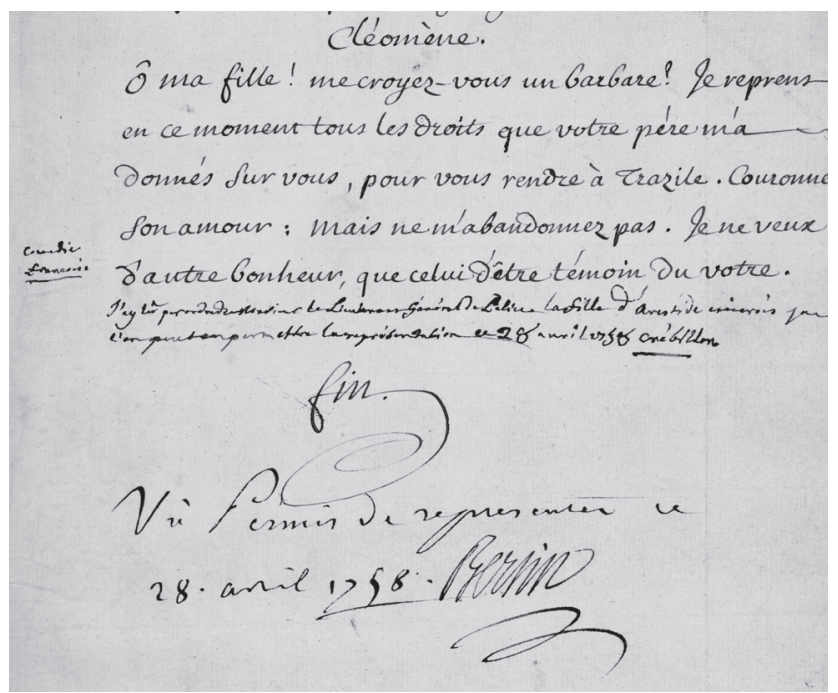

Fig. 7 : Extrait de la dernière page d'une copie destinée à la mise en scène de La Fille d'Aristide, qui contient l'autorisation officielle de la censure au-dessous de la dernière réplique de Cléomène (The Beinecke Rare Book and Manuscript Library, Graffigny Papers, xcvii:263)

- état préliminaire de la dédicace à l'impératrice Marie-Thérèse avec des corrections autographes infrapaginales et sur un emplâtre (xcviii:9-12) (fig. 8).

9. BHVP, 3.399, p. 80 (notice recueillie par D. Trott). 


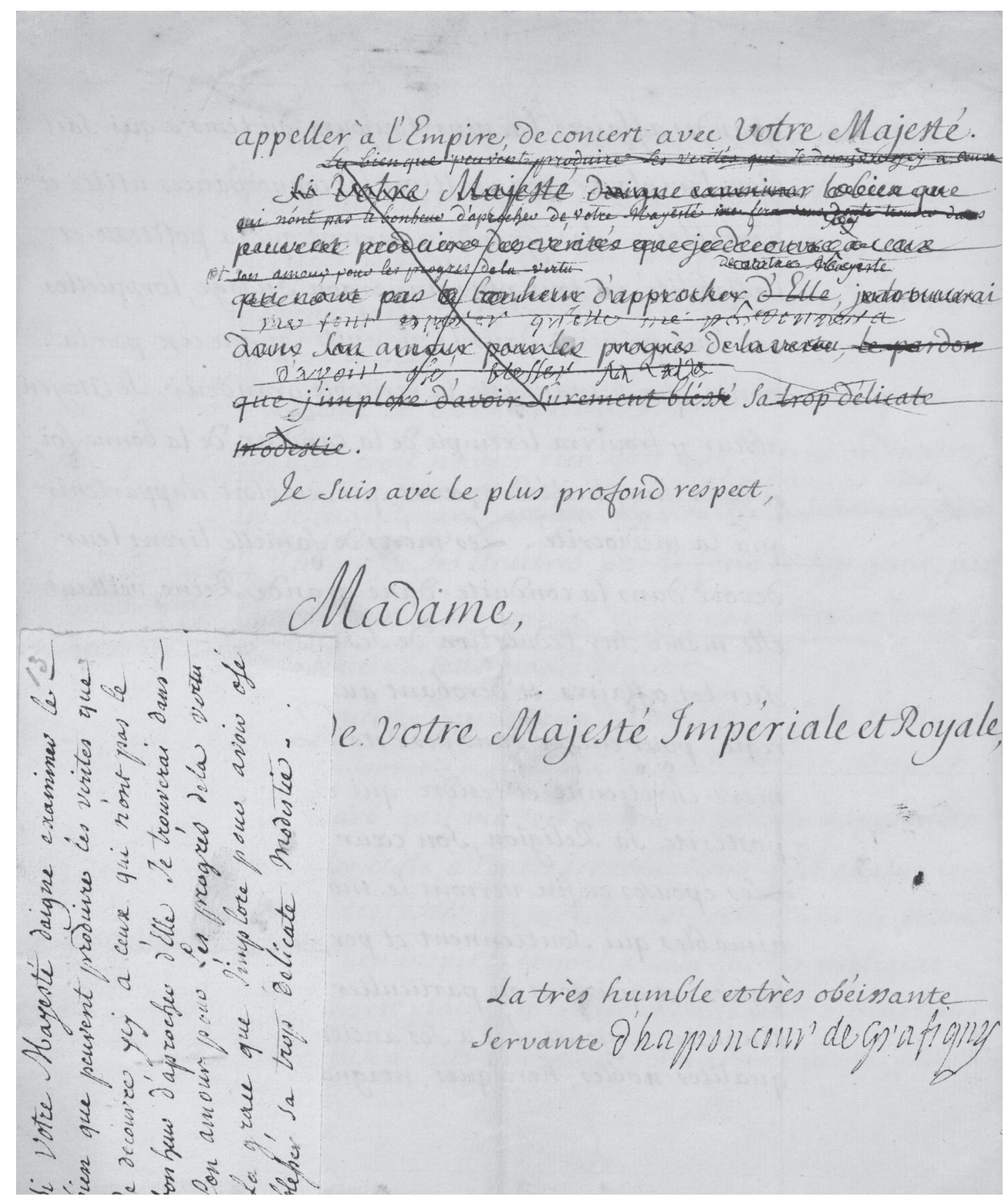

Fig. 8 : Extrait d'un état préliminaire de la dédicace à l'impératrice autrichienne avec des corrections autographes et un emplâtre qui contient la mise au net d'une main non identifiée. La dédicace, considérée trop louangeuse par sa destinatrice, sera abrégée et cet extrait n'y figurera plus

(The Beinecke Rare Book and Manuscript Library, Graffigny Papers, xcviii:12, avec la signature de Mme de Graffigny) 
Christina Ionescu, dix-huitièmiste de formation, enseigne dans le Département de langues et de littératures modernes de l'université Mount Allison au Canada. Spécialiste des rapports texte(s)-image(s), elle a publié des articles sur le livre illustré et sur la culture visuelle de l'Ancien Régime. En tant que Graffigniste, elle s'intéresse notamment aux éditions illustrées des Lettres d'une Péruvienne et à la genèse de La Fille d'Aristide.

cionescu@mta.ca

\section{Résumés}

\section{Dans les coulisses de La Fille d'Aristide: le manuscrit théâtral et les Graffigny Papers sour l'œil de la critique génétique}

Grâce aux soins prodigieux de Mme de Graffigny, de son ami " Panpan » Devaux, de leurs héritiers, de nombreux collectionneurs et bibliothécaires, la plupart des manuscrits littéraires de l'auteure lorraine sont aujourd'hui conservés dans les Graffigny Papers à la Beinecke Library aux États-Unis. Parmi ces documents, le dossier génétique de La Fille d'Aristide, présenté pour la première fois dans le cadre de cet article, s'avère particulièrement riche et intéressant, d'autant plus qu'aucune étude critique n'a été consacrée jusqu'ici à la genèse longue et compliquée de cette pièce de théâtre qui fut un grand échec à la ComédieFrançaise en 1758. Ce sont les aspects suivants qui font l'objet de cette étude : le cheminement avant-textuel de La Fille d'Aristide, les pratiques d'écriture qui sous-tendent le processus de production de l'œuvre, ainsi que les traces des mécanismes sociaux et institutionnels qui influençaient la création littéraire d'une auteure du siècle des Lumières.

Thanks to Mme de Graffigny's great care as well as that of her friend "Panpan" Devaux, of their descendants and of many collectors and librarians, most of this Lorrainer writer's literary manuscripts are now preserved in the Beinecke Library at Yale University. Among these documents, the genetic dossier of $L a$ Fille d'Aristide, presented here for the first time, is particularly rich and interesting, the more so that there has been no study yet of the long and complicated genesis of this play which was a complete failure at the Comédie-Française in 1758. This study focuses on the following aspects: the slow progression of the avant-texte of La Fille d'Aristide, the writing practices underlying the work's production process, as well as the traces of the social and institutional writing mechanisms that influenced the literary creation of a woman writer in the Age of Enlightenment.

Dank der außergewöhnlichen Sorgfalt von Mme de Graffigny, ihres Freundes „Panpan“ Devaux, ihrer Erben sowie zahlreicher Sammler und Bibliothekare sind die meisten der literarischen Handschriften der lothringischen Autorin heute in den Graffigny Papers der Beinecke Library in den Vereinigten Staaten aufbewahrt. Unter diesen Dokumenten befindet sich auch das genetische Dossier von La Fille d'Aristide, das hier zum ersten Mal vorgestellt wird, und sich als besonders reich und interessant erweist, zumal sich bis heute keine einzige Studie mit der langen und komplizierten Genese dieses Theaterstücks befasst hat, das im Jahr 1758 an der Comédie-Française ein großer Misserfolg gewesen war. Folgende Aspekte sind Gegenstand dieser Untersuchung: die Stufen des „Avant-texte“ von La Fille d'Aristide, die dem Produktionsprozess des Werkes zugrunde liegenden Schreibpraktiken, sowie die Spuren sozialer und institutioneller Mechanismen, die das literarische Schaffen einer Autorin im Zeitalter der Aufklärung beeinflussten.
Gracias al cuidado prodigioso de Françoise de Graffigny, de su amigo "Panpan" Devaux, de sus herederos, de numerosos coleccionistas y bibliotecarios, la mayor parte de los manuscritos literarios de la autora, originaria de Lorena, están actualmente custodiados en los Graffigny Papers de la Beinecke Library de los Estados Unidos. Entre esos documentos, el dossier genético de La Fille d'Aristide, presentado por primera vez en el marco de este artículo, se revela como particularmente rico e interesante, tanto más cuanto que, hasta la actualidad, ningún trabajo crítico ha sido dedicado al análisis de la génesis larga y complicada de esta obra de teatro, que fue un gran fracaso en la Comedia Francesa, en 1758. Los siguientes aspectos constituirán nuestro objeto de estudio: el itinerario pre-textual de La Fille d'Aristide, las prácticas de escritura que sustentan el proceso de producción de la obra, así como las huellas de los mecanismos sociales e institucionales que influenciaron la creación literaria de esta autora del Siglo de las Luces.

Grazie alle cure straordinarie di Mme de Graffigny, del suo amico "Panpan" Devaux, dei loro eredi, di numerosi collezionisti e bibliotecari, la maggior parte dei manoscritti letterari dell'autrice lorenese sono oggi conservati nei Graffigny Papers presso la Beinecke Library negli Stati Uniti. Tra questi documenti, il dossier genetico di La Fille d'Aristide, presentato per la prima volta in questo articolo, si mostra particolarmente ricco e interessante, tanto più che nessuno studio critico è mai stato dedicato, fino a oggi, a questa pièce teatrale che subì la sorte di un grave insuccesso alla Comédie-Française nel 1758. Qui si analizzeranno il percorso dell'avantesto dell'opera, le pratiche di scrittura che sottendono il suo processo di produzione, e le tracce dei meccanismi sociali e istituzionali che influenzavano la creazione letteraria di un'autrice del secolo dei Lumi.

Graças ao cuidado da família e de amigos, bem como de coleccionadores e bibliotecários, a maior parte dos manuscritos literários da escritora da Lorraine estão hoje conservados como Graffigny Papers na Beinecke Library (EUA). Entre estes documentos, o dossier genético de La Fille d'Aristide, aqui revelado pela primeira vez, revela-se particularmente rico e interessante, tanto mais que nenhum estudo crítico foi ainda consagrado à longa e complicada génese da peça, mal acolhida na ComédieFrançaise aquando da estreia em 1758. Constam deste estudo os seguintes aspectos: o percurso ante-textual de La Fille d'Aristide, as práticas de escrita que subjazem ao processo de produção da obra, e ainda os vestígios de mecanismos sociais e institucionais que influenciavam a criação literária duma escritora do Século das Luzes. 